\title{
Inequalities and Eigenvalues of Sturm-Liouville Problems Near a Singular Boundary
}

\author{
W. N. EVERITT ${ }^{a, *}$, M. MARLETTA ${ }^{b, \dagger}$ and A. ZETTL ${ }^{c, \ddagger}$

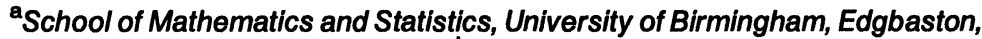 \\ Birmingham B15 2TT, England, UK; ' Department of Mathematics, University \\ of Leicester, University Road, Leicester LEI 7RH, England, UK; ' ${ }^{\circ}$ Department \\ of Mathematics, Northern Illinois University, DeKaLB, IL 60115-2888, USA
}

(Received 3 December 1999; In final form 14 February 2000)

We study the behavior of eigenvalues of Sturm-Liouville problems (SLP) when an endpoint of the underlying interval approaches a singularity.

Keywords and Phrases: Sturm-Liouville; Eigenvalues; Singular endpoint

Mathematics Subject Classifications 1991: Primary: 34B24, 34L10; Secondary: 34L40, 34B20

\section{INTRODUCTION}

In [1] Bailey, Everitt, Weidmann and Zettl studied the approximation of the spectrum of a given singular Sturm-Liouville problem (SLP) with spectra of regular SLP. These regular problems are constructed by truncating the singular interval and choosing appropriate boundary conditions on the truncated interval.

The relationship between spectra of singular and "nearby" regular problems is investigated further in this paper. But here, instead of

\footnotetext{
*Corresponding author. e-mail: w.n.everitt@bham.ac.uk

†e-mail: mm7@le.ac.uk

†e-mail: zettl@math.niu.edu
} 
"going from outside in" we take the "inside out" approach. Given a regular SLP with an endpoint which is close to a singular endpoint, what happens to the eigenvalues as the regular endpoint is moved closer to the singular one? We shall see that the answer depends very strongly on the nature of the spectrum of the singular problem. The singular spectrum is a "strong attractor": it attracts the eigenvalues of the regular problems from the truncated intervals toward itself. This illustrates dramatically the point made by Zettl in [11], and in particular in [12], that in order to understand the behavior of eigenvalues of regular problems one needs to have a perspective which includes the singular case.

Some related results are given in $[2,3,6,7,4,5]$.

For the general theory of linear differential operators see $[9$, Chapter V].

In Section 2 we introduce some notation and summarize the basic results used subsequently. Section 3 contains our main result for the case when the spectrum is not bounded below. Section 4 deals with the case when the spectrum of the singular problem is discrete, and in Section 5 we study the case when the essential spectrum is not bounded below and is non-empty.

\section{NOTATION AND BASIC RESULTS}

In this paper we use the notation and terminology from [1], however for the convenience of the reader, we introduce some of the basic notation here. Consider the equation

$$
-\left(p y^{\prime}\right)^{\prime}+q y=\lambda w y \text { on } J=(a, b) \text { with }-\infty \leq a<b \leq \infty ;
$$

where the coefficients satisfy

$$
p^{-1}, q, w \in L_{\mathrm{loc}}(J, \mathbb{R}) \text { and } p>0, w>0 \text { almost everywhere on } J .
$$

Let $\left\{a_{r}: r \in \mathbb{N}\right\}$ be a decreasing sequence converging to the endpoint $a$, and $\left\{b_{r}: r \in \mathbb{N}\right\}$ an increasing sequence converging to endpoint $b$; here $\mathbb{N}=\{1,2,3, \ldots\}$, the spectral parameter $\lambda \in \mathbb{C}$ and $a<a_{r}<b_{r}<b$; also let $J_{r}=\left(a_{r}, b_{r}\right)$ for all $r \in \mathbb{N}$. 
Under the conditions (2.2) the Eq. (2.1) may be singular at one or both endpoints, but note that it is regular at each of the truncated endpoints $a_{r}, b_{r}$ for $r \in \mathbb{N}$.

Let $S$ be a self-adjoint realization of $(2.1)$ on $(a, b)$, and $S_{r}$ a selfadjoint realization of $(2.1)$ on $\left(a_{r}, b_{r}\right)$ for $r \in \mathbb{N}$. Then $S=S^{*}$ is a selfadjoint operator in the Hilbert function space $L^{2}(J, w)$ and $S_{r}=S_{r}^{*}$ is a self-adjoint operator in the Hilbert space $L^{2}\left(J_{r}, w\right)$ for $r \in \mathbb{N}$. Let $\sigma(S)$, $\sigma\left(S_{r}\right)$ denote the spectra of $S, S_{r}$, respectively. It is well known, see the recent paper [8], that the spectra of both $S$, and $S_{r}$ are not bounded above and that the spectrum of $S_{r}$ is bounded below and discrete. Let $\mathbb{N}_{0}=\{0,1,2, \ldots\}$ and let

$$
\sigma\left(S_{r}\right)=\left\{\lambda_{n}\left(S_{r}\right): n \in \mathbb{N}_{0}\right\}=\left\{\lambda_{n}\left(J_{r}: A_{r}, B_{r}\right): n \in \mathbb{N}_{0}\right\} .
$$

Here $A_{r}, B_{r}$ are the matrices which determine the self-adjoint realization $S_{r}$ on $\left(a_{r}, b_{r}\right)$, for details see [12].

In general, the spectrum of $S$ in the singular case may be quite complicated: there may be no eigenvalues, finitely many, infinitely many, a mixture of eigenvalues and essential spectrum, there may be eigenvalues embedded in the essential spectrum, etc. Let

$$
\sigma_{0}=\inf \sigma_{e}(S),
$$

where $\sigma_{e}(S)$ denotes the essential spectrum of $S$. We distinguish three cases for $\sigma_{e}(S)$ :

$$
\sigma_{0}=-\infty, \quad-\infty<\sigma_{0}<\infty, \quad \sigma_{0}=\infty .
$$

In the first case the essential spectrum is not bounded below, in the second case it is bounded below and not empty, in the third case it is empty, i.e., the spectrum of $S$ is discrete but may or may not be bounded below.

Our results in this paper depend on three Lemmas which we now state.

Lemma 2.1 (Kong, Wu and Zettl). For any fixed $r \in \mathbb{N}$ let $\lambda_{n}^{D}$ denote the $n$-th Dirichlet eigenvalue and let $\lambda_{n}$ denote the $n$-th eigenvalues of any other self-adjoint realization on the interval $\left(a_{r}, b_{r}\right), n \in \mathbb{N}_{0}$. Then

$$
\lambda_{n}^{D} \leq \lambda_{n+2} \leq \lambda_{n+2}^{D} \text { for all } n \in \mathbb{N}_{0} \text {. }
$$

Proof See [5, Section 4, Theorem 4.1]. 
Lemma 2.2 (Bailey, Everitt, Weidmann and Zettl). Let $S$ be a selfadjoint realization on $(a, b)$ and $S_{r}^{i}$ its induced restriction on $\left(a_{r}, b_{r}\right)$. Then the sequence $\left\{S_{r}^{i}: r \in \mathbb{N}\right\}$ is spectral included for $S$, i.e., given any $\lambda \in \sigma(S)$ there exists $n(r, \lambda) \in \mathbb{N}_{0}$ for each $r \in \mathbb{N}$ such that

$$
\left\{\lambda_{n(r, \lambda)}\left(S_{r}^{i}\right)\right\} \rightarrow \lambda, \text { as } r \rightarrow \infty
$$

Proof See [1].

Lemma 2.3 (Bailey, Everitt, Weidmann and Zettl). Let the hypotheses and notation of Lemma 2.2 hold.

1. If $\sigma(S)$ is bounded below, then the sequence $\left\{S_{r}^{i}: r \in \mathbb{N}\right\}$ is spectral exact for $S$ below $\sigma_{0}(S)$, this means that if

$$
\left\{\lambda_{n(r, \lambda)}\left(S_{r}^{i}\right)\right\} \rightarrow \lambda, \text { as } r \rightarrow \infty,
$$

and $\lambda<\sigma_{0}(S)$, then $\lambda \in \sigma(S)$.

2. If each endpoint of $(a, b)$ is, independently, either $R$ or $L C$, then $\left\{S_{r}^{i}: r \in \mathbb{N}\right\}$ is spectral exact for $S$, regardless of whether the spectrum of $S$ is bounded below or not.

3. If $\sigma(S)$ is bounded below and discrete, then

$$
\left\{\lambda_{n}\left(S_{r}^{i}\right)\right\} \rightarrow \lambda_{n}, \text { as } r \rightarrow \infty, \text { for each } n \in \mathbb{N}_{0} .
$$

Proof See [1].

\section{THE SPECTRUM IS UNBOUNDED BELOW}

Our first main result deals with the case when the singular spectrum is not bounded below and shows the effect this has on the eigenvalues of any self-adjoint extension of the "nearby" regular problems.

Theorem 3.1 Assume that $\sigma(S)$ is not bounded below. Suppose $S_{r}$ is any self-adjoint realization on the interval $\left(a_{r}, b_{r}\right)$ with spectrum $\sigma\left(S_{r}\right)=\left\{\lambda_{n}\left(S_{r}\right): n \in \mathbb{N}_{0}\right\}$. ( $S_{r}$ is, in general, determined by different boundary conditions for different $r$.) Then

$$
\left\{\lambda_{n}\left(S_{r}\right)\right\} \rightarrow-\infty \text { as } r \rightarrow \infty, \text { for each } n \in \mathbb{N}_{0} .
$$


Proof Denote the eigenvalues of the induced restriction $S_{r}$ by $\lambda_{n}^{i}=$ $\lambda_{n}\left(S_{r}\right)$ for all $n \in \mathbb{N}_{0}$. By Lemma 2.1 it is sufficient to show that each sequence of Dirichlet eigenvalues tends to $-\infty$. It is well known that each Dirichlet eigenvalue is a decreasing function of the increasing length of the interval. (This follows from the variational characterization of the Dirichlet eigenvalues; for a different proof see [7]. This latter proof of Kong and Zettl makes it clear why each eigenvalue is a decreasing function of the increasing length of the interval in the case of Dirichlet boundary conditions and, in general, for no other set of separated self-adjoint boundary conditions.) Hence for each $n \in \mathbb{N}_{0}$ the limit

$$
\left\{\lambda_{n}^{D}\left(a_{r}, b_{r}\right)\right\} \rightarrow l_{n} \text { as } r \rightarrow \infty
$$

exists. We claim: $l_{n}=-\infty$. Suppose $l_{n}>-\infty$. Choose $\mu_{k} \in \sigma(S)$ such that

$$
\mu_{n+3}<\mu_{n+2}<\mu_{n+1}<\cdots<\mu_{1}<\mu_{0}<l_{n} .
$$

By spectral inclusion of Lemma 2.2, for each $\mu_{k}$ there exists an index sequence $n\left(r, \mu_{k}\right)$ such that

$$
\left\{\lambda_{n\left(r, \mu_{k}\right)}^{i}\right\} \rightarrow \mu_{k} .
$$

From this and Lemma 2.1 it follows that $n\left(r, \mu_{k}\right) \geq k$. Again from Lemma 2.1 we get that $\lambda_{n\left(r, \mu_{n+3}\right)-2}^{D}<\lambda_{n}^{D}$, which is a contradiction since $n\left(r, \mu_{n+3}\right)-2 \geq n$. Thus $\left\{\lambda_{n}^{D}\left(a_{r}, b_{r}\right)\right\} \rightarrow-\infty$ as $r \rightarrow \infty$ for each $n \in \mathbb{N}_{0}$, and the conclusion follows since $\lambda_{n}^{D}\left(a_{r}, b_{r}\right) \geq \lambda_{n}$ for the $n$-th eigenvalue $\lambda_{n}$ of any other self-adjoint boundary condition.

\section{DISCRETE SPECTRUM}

The next result is part of Lemma 2.3; we state it here to highlight it and because it contrasts with Theorem 3.1 of Section 3. We remark further on this contrast below.

Theorem 4.1 Assume that $\sigma(S)$ is bounded below and discrete with spectrum $\sigma(S)=\left\{\lambda_{n}(S): n \in \mathbb{N}_{0}\right\}$. Suppose $S_{r}^{i}$ is the induced restriction of $S$ on the interval $\left(a_{r}, b_{r}\right)$ as defined in [1] with spectrum 


$$
\begin{aligned}
\sigma\left(S_{r}^{i}\right)=\left\{\lambda_{n}^{i}\left(S_{r}\right): n \in \mathbb{N}_{0}\right\} . \text { Then } \\
\\
\left\{\lambda_{n}^{i}\left(S_{r}\right)\right\} \rightarrow \lambda_{n}(S) \text { as } r \rightarrow \infty \text { for each } n \in \mathbb{N}_{0} .
\end{aligned}
$$

Proof This is part (3) of Lemma 2.3.

Remark 4.1 Note the contrast between Theorem 3.1 and Theorem 4.1. This illustrates dramatically the enormous influence that the spectrum of a singular problem has on the eigenvalues of nearby regular problems. This point becomes even more interesting when viewed in terms of the asymptotic behavior of the eigenvalues: For any fixed $r$ and any fixed self-adjoint realization $S_{r}$ on the interval $\left(a_{r}, b_{r}\right)$ the eigenvalues are asymptotic to $n^{2}$ as $n \rightarrow \infty$; more precisely, see [10],

$$
\frac{\lambda_{n}\left(S_{r}\right)}{n^{2}} \rightarrow e=\pi^{2}\left(\int_{J_{r}} \sqrt{\frac{w}{p}}\right)^{-2} .
$$

\section{THE ESSENTIAL SPECTRUM IS BOUNDED BELOW AND NON-EMPTY}

When $\sigma(S)$ is bounded below and $-\infty<\sigma_{0}(S)<\infty$ then the spectrum of regular problems on truncated intervals is affected by both the essential spectrum and by the eigenvalues below the essential spectrum. The next result details the effect of the eigenvalues below the essential spectrum and the very special and strong attraction of the starting point of the essential spectrum $\sigma_{0}(S)$; for illustrations of the effect of the first few spectral bands and gaps see the paper by Zettl [11].

THEOREM 5.1 Assume that the spectrum of $S$ is bounded below and $-\infty<\sigma_{0}(S)<\infty$. Suppose $S_{r}^{i}$ is the induced restriction of $S$ on the interval $\left(a_{r}, b_{r}\right)$ as defined in [1] with spectrum $\sigma\left(S_{r}^{i}\right)=\left\{\lambda_{n}^{i}\left(S_{r}\right): n \in \mathbb{N}_{0}\right\}$. Then

1. If $S$ has no eigenvalue below $\sigma_{0}(S)$, we have

$$
\left\{\lambda_{n}^{i}\left(S_{r}\right)\right\} \rightarrow \sigma_{0}(S) \text { as } r \rightarrow \infty \text { for each } n \in \mathbb{N}_{0} .
$$


2. If $S$ has exactly $k$ eigenvalues below $\sigma_{0}(S)$, say $\lambda_{0}, \lambda_{1}, \ldots, \lambda_{k-1}$, $k \in \mathbb{N}$, we have

$$
\left\{\lambda_{j}^{i}\left(S_{r}\right)\right\} \rightarrow \lambda_{j} \text { as } r \rightarrow \infty ; \text { for } j=0,1, \ldots, k-1 ;
$$

and

$$
\left\{\lambda_{n}^{i}\left(S_{r}\right)\right\} \rightarrow \lambda_{n} \text { as } r \rightarrow \infty \text { for each } n=k, k+1, k+2, \ldots
$$

3. If $S$ has an infinite number of eigenvalues below $\sigma_{0}(S)$, say $\left\{\lambda_{n}: n \in \mathbb{N}_{0}\right\}$ then

$$
\left\{\lambda_{n}^{i}\left(S_{r}\right)\right\} \rightarrow \lambda_{n} \text { as } r \rightarrow \infty \text { for each } n \in \mathbb{N}_{0} .
$$

Proof Proceed as follows:

1. To prove (1) choose a strictly decreasing sequence $\left\{\mu_{k} \in \sigma(S)\right.$ : $k \in \mathbb{N}\}$ converging to $\sigma_{0}(S)$; such a sequence exists since the essential spectrum is closed. Now argue as in the Proof of Theorem 3.1 with $\sigma_{0}(S)$ playing the role of $-\infty$.

2. To prove (2) and (3) see the arguments in [1, Theorems 5.3 and 6.4]; the proofs required are similar to those given for these quoted theorems, although the latter cover the case of a discrete spectrum.

Remark 5.1 The eigenvalues below $\sigma_{0}(S)$, if there are any, are approximated by $\lambda_{n}^{i}\left(a_{r}, b_{r}\right)$ for fixed $n=0,1,2, \ldots, k-1$ in the case of exactly $k$ eigenvalues and for all $n$ if there are an infinite number of such eigenvalues. By spectral inclusion every point $\lambda$ of the spectrum $\sigma(S)$ can be approximated by some sequence of eigenvalues, $\left\{\lambda_{n(r, \lambda)}\right\}$ with $n(r, \lambda) \in \mathbb{N}$. If $\lambda \geq \sigma_{0}(S)$ then this sequence of indices $\{n(r, \lambda)\}$ cannot be constant, in fact it cannot be bounded. Thus there is extensive "index jumping" in process for the sequence $\left\{\lambda_{n}(r, \lambda)\right\}$ for each such $\lambda$.

Theorem 5.1 describes the behavior of the eigenvalues for the inherited boundary conditions on the truncated intervals $\left(a_{r}, b_{r}\right)$. What about the eigenvalues of other self-adjoint problems from these intervals? By combining Theorem 5.1 with Lemma 2.1 we get information about the location of the eigenvalues of arbitrary selfadjoint realizations from the truncated intervals. 
THEOREM 5.2 Assume that the spectrum of $S$ is bounded below and $-\infty<\sigma_{0}(S)<\infty . J_{r}=\left(a_{r}, b_{r}\right)$ with either separated or coupled boundary conditions; these realizations may be different for different $r$. Then

1. If $S$ has no eigenvalue below $\sigma_{0}(S)$, we have

$$
\left\{\lambda_{n}\left(a_{r}, b_{r}\right)\right\} \rightarrow \sigma_{0}(S) \text { as } r \rightarrow \infty \text { for each } n \in \mathbb{N}_{0} .
$$

2. If $S$ has exactly $k$ eigenvalues below $\sigma_{0}(S), k \in \mathbb{N}$, then

$$
\left\{\lambda_{n+2}\left(a_{r}, b_{r}\right)\right\} \rightarrow \sigma_{0}(S) \text { as } r \rightarrow \infty \text { for all } n \geq k \text {. }
$$

3. If $S$ has an infinite number of eigenvalues below $\sigma_{0}(S)$, say $\left\{\lambda_{n}: n \in \mathbb{N}_{0}\right\}$ then the eigenvalues $\lambda_{n}\left(a_{r}, b_{r}\right)$ "bunch up to the left of $\sigma_{0}(S)$ ". Let $l_{n}=\lim _{r \rightarrow \infty} \lambda_{n}^{D}\left(a_{r}, b_{r}\right), n \in \mathbb{N}_{0}$. Then

$$
\lambda_{n} \leq l_{n} \leq \lambda_{n+2}, n \in \mathbb{N}_{0},\left\{\lambda_{n}\right\} \rightarrow \sigma_{0}(S) .
$$

Proof Parts (1) and (2) follow from Theorem 5.1 and Lemma 2.1. To obtain the inequality of part (3) let $r \rightarrow \infty$ in the inequality

$$
\lambda_{n}^{i}\left(a_{r}, b_{r}\right) \leq \lambda_{n}^{D}\left(a_{r}, b_{r}\right) \leq \lambda_{n+2}^{i}\left(a_{r}, b_{r}\right),
$$

and recall the well known fact that, since the spectrum is bounded below, the eigenvalues below $\sigma_{0}(S)$ can accumulate only at $\sigma_{0}(S)$.

Remark 5.2 If the spectrum of one self-adjoint extension is bounded below then the spectrum of every self-adjoint extension is bounded below. Thus in each of the above theorems where we assume that $\sigma(S)$ is or is not bounded below, this is not an assumption about a particular operator $S$. The minimal operator of $(2.1)$ is bounded below if and only if the spectrum of every self-adjoint realization of (2.1) is bounded below.

\section{References}

[1] Bailey, P. B., Everitt, W. N., Weidmann, J. and Zettl, A. (1993). 'Regular approximation of singular Sturm-Liouville Problems'. Results in Mathematics, 23, 3-22.

[2] Everitt, W. N., Möller, M. and Zettl, A., 'Discontinuous dependence of the $n$-th Sturm-Liouville eigenvalue'. (General Inequalities $\gamma$; Proceedings of International Conference on General Inequalities 7, Ober-wolfach, 1994. (International Series of Numerical Mathematics, 123 (1997), 147-150; Birkhauser-Verlag, Basel; Edited by Bandle, C., Everitt, W. N., Losonczi, L. and Walter, W.). 
[3] Everitt, W. N., Möller, M. and Zettl, A. (1999). Sturm-Liouville problems and discontinuous eigenvalues. Proc. Roy. Soc. Edinb. (A), 129, 707-716.

[4] Kong, Q., Wu, H. and Zettl, A. (1997). 'Dependence of eigenvalues on the problem'. Mathematische Nachrichten, 188, 173-201.

[5] Kong, Q., Wu, H. and Zettl, A. (1999). 'Dependence of the $n$-th Sturm-Liouville eigenvalue on the problem'. J. Diff. Equations, 156, 328-354.

[6] Kong, Q. and Zettl, A. (1996). 'Dependence of eigenvalues of Sturm-Liouville problems on the boundary'. J. Diff. Equations, 126, 389-407.

[7] Kong, Q. and Zettl, A. (1996). 'Eigenvalues of regular Sturm-Liouville problems'. J. Diff. Equations, 131, 1-19.

[8] Möller, M. (1999). 'On the unboundedness below of the Sturm-Liouville operator'. Proc. Roy. Soc. Edinburgh (A), 129, 1011-1015.

[9] Naimark, M. A., Linear differential operators: II. (Ungar, New York, 1968. Translated from the second Russian Edition, 1966).

[10] Niessen, H.-D. and Zettl, A. (1992). 'Singular Sturm-Liouville problems: The Friedrichs extension and comparison of eigenvalues'. Proc. London Math. Soc., 64, $545-578$.

[11] Zettl, A. (1993). 'Computing continuous spectrum'. Proceedings of the International Symposium on Trends and Developments in Ordinary Differential Equations, pp. 393-406. (World Scientific Publishing Company, Singapore, 1993; Edited by Alavi, Y. and Hseih, P.-F.).

[12] Zettl, A., 'Sturm-Liouville problems'. Spectral theory and computational methods of Sturm-Liouville problems: Lecture Notes in Pure and Applied Mathematics, 191, 251-283. (Marcel Dekker, Inc., New York, 1997). 